\title{
CHEMICAL-PHYSICAL CHANGES IN CELL MEMBRANE MICRODOMAINS OF BREAST CANCER CELLS AFTER OMEGA-3 PUFA INCORPORATION
}

Paola A. Corsetto, Andrea Cremona, Gigliola Montorfano, Ilaria E. Jovenitti, Francesco Orsini, Paolo Arosio, Angela M. Rizzo

$\S$ Corresponding Author Angela Maria Rizzo, Dipartimento di Scienze Molecolari Applicate ai Biosistemi, Università degli Studi di Milano, Via D. Trentacoste 2, 20134 Milan, Italy. Phone: +39 02503 15789.Fax: $+390250315775$

E-mail: angelamaria.rizzo@unimi.it paola.corsetto@unimi.it gigliola.montorfano@unimi.it ilaria.jovenitti@unimi.it francesco.orsini@unimi.it paolo.arosio@unimi.it andrea.cremona@unimi.it

Abbreviations: PLs (phospholipids), FA (fatty acids), PUFA (polyunsaturated fatty acids), MUFA (monounsaturated fatty acids), SFA (saturated fatty acids), DHA (docosahexaenoic acid), EPA (eicosapentaenoic acid), AA (arachidonic acid), PE (phosphatidylethanolamine), PI (phosphatidylinositol), PC (phosphatidylcholine), PS (phosphatidylserine), SM (sphingomyelin), Chol (cholesterol). 


\section{Abstract}

Epidemiologic and experimental studies suggest that dietary fatty acids influence the development and progression of breast cancer. However no clear data are present in literature that could demonstrate how n-3 PUFA can interfere with breast cancer growth. It is suggested that these fatty acids might change the structure of cell membrane, especially of lipid rafts. During this study we treated MCF-7 and MDA-MB-231 cells with AA,EPA and DHA to asses if they are incorporated in lipid raft phospholipids and are able to change chemical an physical proprieties of these structures. Our data demonstrate that PUFA and their metabolites are inserted with different yield in cell membrane microdomains and are able to alter fatty acid composition without decreasing the total percentage of saturated fatty acids that characterize these structures. In particular in MDA-MB-231 cells, that displays the highest content of Chol and saturated fatty acids, we observed the lowest incorporation of DHA, probably for sterical reasons; nevertheless DHA was able to decrease Chol and SM content. Moreover PUFA are incorporated in breast cancer lipid rafts with different specificity for the phospholipids moiety, in particular PUFA are incorporated in PI, PS and PC phospholipids that may be relevant to the formation of PUFA metabolites (prostaglandins, prostacyclins, leukotrienes, resolvines and protectines) of phospholipids deriving second messengers and signal transduction activation. The bio-physical changes after n-3 PUFA incubation have also been highlighted by Atomic Force Microscopy. In particular, for both cell lines the DHA treatment produced a decrease of the lipid rafts in the order of about $20 \%-30 \%$. It is worth noticing that after DHA incorporation lipid rafts exhibit two different height ranges. In fact, some lipid rafts have a higher height of 6-6.5 nm. In conclusion n-3 PUFA are able to modify lipid raft biochemical and biophysical features leading to decrease of breast cancer cell proliferation probably through different mechanisms related to acyl chain length and unsaturation. While EPA may contribute to cell apoptosis mainly through decrease of AA concentration in lipid raft phospholipids, DHA may change the biophysical properties of lipid rafts decreasing the content of cholesterol and probably a redistribution of key proteins. 


\section{Introduction}

The term omega-3 fatty acids (n-3 or $\omega-3$ ) refers to a class of polyunsaturated fatty acids (PUFA) having the last double bond in the n-3 position. The main dietary sources of eicosapentaenoic (EPA, 20:5n-3) and docosahexaenoic acid (DHA, 22:6n-3) are cold-water fish oils. Epidemiological studies have suggested that an increased n-3 PUFA intake might be associated with a reduced breast cancer incidence in humans [1-3]. A number of mechanisms have been proposed for the anticancer actions of n-3 PUFA, including suppression of neoplastic transformation, inhibition of cell proliferation, enhancement of apoptosis, and antiangiogenic action. Most of these mechanisms have been directly or indirectly linked to their inhibition of the production of eicosanoids from n-6 PUFA. In addition, it has also been suggested that these fatty acids might change the fluidity and structure of cell membrane, especially of lipid rafts. The plasma membrane is involved in almost all aspects of cell biology, including morphogenesis, proliferation, migration, invasion, transformation, differentiation, secretion and apoptosis. Numerous experimental data indicate that the presence of PUFA in the membrane bilayer might determine dramatic changes in physicalchemical properties [4-5], a significant lowering of cholesterol solubility [6], and changes in the activity of transmembrane proteins such as the G-protein coupled membrane receptors [7-8]. In fact the structure and dynamics of these polyunsaturated chains at the molecular level are profoundly different from their saturated counterpart, with very short reorientational correlation times and extremely low chain order.

In the past two decades, a growing number of data indicate that membrane lipids exist in gel, liquidordered or liquid-disordered states, depending on the complex environment of the cell. Subsequently, a lipid raft theory evolved in which liquid-ordered microdomains or lipid rafts, are floating over the "sea" of bulk membrane, which is in a liquid-disordered state. The preponderance of saturated hydrocarbon chains in sphingolipids allows for tight cholesterol interaction, thereby forming a "packed" liquid-ordered phase [9-10]. Lipid rafts can be classified as morphologically featureless, detergent-resistant membranes (DRMs) due to their insolubility in cold non ionic 
detergents [11]. These domains are characterized physicochemically by a relative rigidity and reduced fluidity compared with the surrounding plasma membrane, which is in part caused by their cholesterol content [12-14]. Lipid rafts are highly dynamic and may rapidly assemble and disassemble, leading to a dynamic segregation of proteins $[12,15]$. In fact rafts localization is shown to modulate a variety of proteins, such as receptor activities and therefore signal transduction $[16,17]$. Cholesterol alterations, which affect raft structure, also might alter the receptor function [16]. For example, it is now known that $\mathrm{T}$ cell intracellular signalling cascades, endocytosis, protein trafficking, and cell-cell communication are modulated in part by altering the lipid-protein composition of the bulk membrane and specialized lipid microdomains [18, 19].

In the present paper, we analyzed the incorporation of PUFA in cancer cell lipid rafts by HPLC/GC analysis of raft phospholipid fatty acid composition. In addition, morpho-dimensional changes in lipid rafts have been visualized and evaluated by atomic force microscopy (AFM) studying purified membrane samples both before and after the n-3 PUFA treatment. AFM technique allows obtaining three-dimensional images of the surface topography of biological specimens (i.e. lipid microdomains) at nanometer resolution in a physiological-like environment thus providing structural/functional insights that cannot be obtained with more conventional approaches.

\section{MATERIALS AND METHODS}

\subsection{Materials}

EPA (cis-5,8,11,14,17-eicosapentaenoic acid sodium salt), DHA (cis-4,7,10,13,16,19docosahexaenoic acid sodium salt) and AA (cis-5,8,11,14-arachidonic acid sodium salt) were purchased from Sigma-Aldrich, US. PUFA are dissolved in ethanol and stored at $-80^{\circ} \mathrm{C}$ under $\mathrm{N}_{2}(\mathrm{~g})$. The rabbit polyclonal anti-flotillin-1 and the mouse monoclonal anti-clathrin heavy chain (HC) antibody were purchased from Santa Cruz Biotechnology Inc., Santa Cruz, CA, USA. Bound primary antibody is visualized by proper secondary horseradish peroxidase (HRP)-linked antibody, purchased from Santa Cruz Biotechnology Inc., Santa Cruz, CA, USA. 


\subsection{Cell lines and culture conditions}

Human breast cancer cells MDA-MB-231 (ER-negative) and MCF7 (ER-positive) were kindly provided by IST (Italian National Cancer Research Institute, Genova Italy, laboratory of Molecular Mutagenesis and DNA repair, Dr. Degan). Both cell lines derive from human mammary adenocarcinoma; MCF7 line retains several characteristics of differentiated mammary epithelium including ability to process estradiol via cytoplasmic estrogen receptors. The MDA-MB-231 cells over-express the receptor of epidermal growth factor (EGFR).

Both cell lines are routinely maintained in DMEM medium (Gibco-BRL, Life Tecnologies Italia srl, Italy) supplemented with $10 \%$ fetal bovine serum (FBS), $100 \mathrm{U} / \mathrm{ml}$ penicillin, $100 \mathrm{mg} / \mathrm{ml}$ streptomycin and $2 \mathrm{mM}$ glutamine.

During treatments the culture medium contains $10 \%$ fetal bovine serum (FBS) (medium for treatments). Cells are grown at $37^{\circ} \mathrm{C}$ in $5 \% \mathrm{CO}_{2}$ at $98 \%$ relative humidity.

\subsection{Lipid rafts isolation}

Previous experiments with MDA-MB-231 and MCF7 cells allowed us to determine the concentrations of EPA $(230 \mu \mathrm{M})$ and DHA $(200 \mu \mathrm{M})$, required to inhibit cell growth by $20 \%-30 \%$. In both cell lines AA is used at the concentration of $200 \mu \mathrm{M}$, without inducing cell death [20].

Cells are seeded at $1.5 \times 10^{4}$ cells $/ \mathrm{cm}^{2}$ for MDA-MB-231 and $3 \times 10^{4}$ cells $/ \mathrm{cm}^{2}$ for MCF7 in $18 \mathrm{ml}$ of medium containing $10 \% \mathrm{v} / \mathrm{v}$ FBS for $48 \mathrm{hrs}$ to adhere. After $48 \mathrm{hrs}$, medium is replaced with fresh medium containing the experimental fatty acids (AA, EPA or DHA). Cells are treated for 72 hrs. Experiments include control cells, which are not exposed to any exogenous fatty acids, but to equal concentration of ethanol. After treatment cells are harvested by scrapering in phosphate-buffered saline containing $0.4 \mathrm{mM} \mathrm{Na} \mathrm{VO}_{4}$. Cells are then centrifuged and suspended in $1.4 \mathrm{ml}$ lysis buffer (1\% Triton X-100, $10 \mathrm{mM}$ Tris buffer, $\mathrm{pH}$ 7.5, $150 \mathrm{mM} \mathrm{NaCl}, 5 \mathrm{mM}$ EDTA, $1 \mathrm{mM} \mathrm{Na} \mathrm{VO}_{4}, 1 \mathrm{mM}$ phenylmethylsulfonyl fluoride, 75 milliunits/ml aprotinin), allowed to stand on ice for 20 min, and finally treated with Dounce homogenizer (10 strokes, tight).

To isolate lipid rafts cell lysate is centrifuged ( $5 \mathrm{~min}$ at 1,300xg) and the supernatant is transferred 
to eppendorf tubes. $1 \mathrm{ml}$ of lysate is mixed in ultracentrifuge tubes (Beckman Coulter) with equal volume of $85 \%$ sucrose (w/v) in $10 \mathrm{mM}$ Tris buffer, $\mathrm{pH} 7.5,150 \mathrm{mM} \mathrm{NaCl}, 5 \mathrm{mM}$ EDTA, and 1 $\mathrm{mM} \mathrm{Na}_{3} \mathrm{VO}_{4}$, and then overlaid with $5.5 \mathrm{ml}$ of $30 \% \mathrm{w} / \mathrm{v}$ sucrose (in the previous buffer) and $4 \mathrm{~mL}$ of $5 \% \mathrm{w} / \mathrm{v}$ sucrose. Tubes are centrifuged at 200,000xg; $17 \mathrm{hrs}$ at $4^{\circ} \mathrm{C}$ (Optimal LE-80K Ultracentrifuge, Beckman Coulter). After centrifugation 11 fractions are collected; to confirm the location of lipid rafts, the content of cholesterol (Chol), sphingomyelin (SM), gangliosides, flotillin1 and clathrin HC is determined in each fraction by HP-TLC and Western Blot. Lipid rafts are usually isolated in 5 and 6 fractions.

\subsection{Lipid extraction}

Fractionated cell membranes were extracted with three different chloroform/methanol mixtures 1:1, 1:2, 2:1 (v/v) and partitioned chloroform/methanol/water, 47:48:1, v/v/v and then with water. The organic phase, after partitioning, is dried and resuspended in chloroform/methanol (2:1) for the analysis of phospholipids, gangliosides and cholesterol content.

\subsection{Characterization of Lipid Rafts}

Free Chol and SM were separated by HP-TLC using silice gel HPTLC plates (Merck, Darmstadt, Germany). Chromatography was performed in hexane/ether/glacial acetic acid (90:10:1 by volume) and with chloroform/methanol/glacial acetic acid/water (60:45:4:2 by volume), respectively. Chol was visualized with a solution of copper sulphate in phosphoric acid at $180{ }^{\circ} \mathrm{C}$, while SM with anisaldehyde in acetic acid and sulphuric acid at $120^{\circ} \mathrm{C}$. Chol and SM standards were spotted on the same plate.

While polar glycosphingolipids, contained in the upper phase, were always separated by HP-TLC using silice gel plates. Chromatography was performed in chloroform/methanol/0.25\% aqueous $\mathrm{CaCl}_{2}(5: 4: 1 \mathrm{v} / \mathrm{v} / \mathrm{v})$. Plates were the air dried and gangliosides visualized with resorcinol at $120^{\circ} \mathrm{C}$. Ganglioside standards were spotted on the same plate. 
For protein characterization of lipid rafts, all fractions were resolved on sodium dodecyl sulphatepolyacylamide gel electrophoresis (SDS-PAGE) and incubated with different antibodies to raft (flotillin-1) and non-raft (clathrin HC) markers [21].

Equal volumes of all fractions were resuspended in SDS loading buffer and boiled at $100^{\circ} \mathrm{C}$ for 5 min to avoid to protein aggregates. Protein contents were quantified by Lowry assay [22]. Samples were run on SDS-PAGE, transferred to polyvinylidene fluoride (PVDF) transfer membranes. Membranes were first incubated with rabbit polyclonal anti-flotillin-1 antibody (1:200) and then incubated with the corresponding horseradish peroxidise (HRP)-linked secondary antirabbit antibody. Afterward, the same membrane was successively reblotted with the antibody specifically directed to clathrin $\mathrm{HC}$, followed by incubation with the corresponding HRP-linked secondary antibody.

Finally, the specific antibody signals were visualized by using the enhanced chemiluminescence (ECL-PLUS, Perkin Elmer, USA ), followed by autoradiography.

\subsection{Analysis of different classes of lipid rafts PLs and cholesterol}

Purification and quantitative analysis of membrane phospholipids and cholesterol is obtained using a HPLC-ELSD system (Jasco, Japan; Sedex SEDERE, FR) equipped with a LiChrospher Si 60 column (LiChroCART 250-4, Merck, Darmstadt, Germany).

The chromatographic separation is carried out as previously described [20]. Evaporative Light Scattering Detector (ELSD) is used to detect and quantify separated PL species, in comparison with calibration standard curves.

After elution, samples are splitted in two aliquots. The ratio is 1:9, i.e. one part to the detector and nine parts are collected by Gilson Fraction Collector Model 201, in order to separate the different phospholipid classes for further GC analysis.

After separation, each phospholipid class is analysed for fatty acid composition by GC in following conditions. 
Fatty acid methyl esters are obtained by transesterification with sodium methoxide in methanol $3.33 \% \mathrm{w} / \mathrm{v}$ and injected into Agilent (Agilent Technologies 6850 Series II) gaschromatograph, equipped with a flame ionization detector (FID) under the following experimental conditions: capillary column: AT Silar length $30 \mathrm{~m}$, film thickness $0.25 \mu \mathrm{M}$. Gas carrier: helium, temperature Injector $250^{\circ} \mathrm{C}$, detector $275^{\circ} \mathrm{C}$, oven $50^{\circ} \mathrm{C}$ for $20 \mathrm{~min}$, rate of $10^{\circ} \mathrm{C} \mathrm{min}{ }^{-1}$ until $200^{\circ} \mathrm{C}$ for $20 \mathrm{~min}$.

\subsection{AFM imaging}

For AFM imaging, purified membrane samples, namely the fraction 5 obtained by the ultracentrifugation process described above, were diluted 1:30 in a adsorption buffer $(150 \mathrm{mM} \mathrm{KCl}$, $25 \mathrm{mM} \mathrm{MgCl} 2,10 \mathrm{mM}$ Tris/ $\mathrm{HCl} \mathrm{pH}$ 7.5). $50 \mu \mathrm{L}$ of the solution is floated on a freshly cleaved mica leaf and let adhered for 10 minutes. Then, sample is gently rinsed three times with a recording buffer $(150 \mathrm{mM} \mathrm{KCl}, 10 \mathrm{mM}$ Tris/HCl $\mathrm{pH}$ 7.5) to remove membranes that have not been strongly adsorbed to the mica support. A drop of $70 \mu \mathrm{l}$ of recording buffer is placed on the mica support before AFM imaging.

AFM imaging is performed using a Multimode Nanoscope IIId (Veeco, Santa Barbara, CA, USA) equipped with a $12 \mu \mathrm{m}$ scanner and sharpened $\mathrm{Si}_{3} \mathrm{~N}_{4}$ cantilevers with a constant spring of $0.06 \mathrm{~N} / \mathrm{m}$ and a $10 \mathrm{~nm}$ curvature radius (Veeco, Santa Barbara, CA, USA). The AFM is operated in contact mode in constant force conditions. The set point is manually adjusted and kept as low as possible to obtain the best resolution while the total force applied on the sample during the imaging is approximately $300 \mathrm{pN}$ as measured by force-distance curve. The $512 \times 512$ pixel $^{2}$ images are recorded at typical scan frequencies of 4-6 Hz. AFM images are flattened using the SPMLab NT Ver. 6.0.2 software (Veeco, Santa Barbara, CA, USA).

\section{RESULTS}

\subsection{Characterization of Lipid Rafts from human breast cancer cells}

We first tested the purity of lipid rafts isolated from human breast cancer cells, MDA-MB-231 and MCF7 cells, using lipid and protein raft and non-raft markers. 
The purification of microdomains was demonstrated by the enrichment of specific phospholipids, especially sphingomyelin, cholesterol and gangliosides, in particular $\mathrm{GM}_{1}$. Lipid rafts are usually isolated in fractions 5 and 6 in all treated and untreated cells (Fig. 1).

Thus, specific antibody directed to lipid raft marker, flotillin-1, was used to identified raft-enriched fractions, observing that this marker was mostly present in fractions 5 and 6 (Fig. 1). The same membranes were then used to reblot with antibody directed to clathrin HC, a protein highly represented in no raft fractions of plasma membrane [21], observing that this antibody recognized some bands especially in fraction 11 (Fig. 1). No significant differences are visualized between the different treatments and control cells.

3.2. Incorporation of PUFA in lipid rafts of breast cancer cells After raft isolation we analyzed by gas chromatography the fatty acid profile of total cell lipid extract, of cell phospholipids and of the raft fractions obtained from both treated and untreated cells.

Even if no radiolabel compounds were used, we calculated the percentage of PUFA incorporated into cell lipids. Data are reported in Table 1; it is possible to observe that MCF-7 incorporate an higher percentage of PUFA compared to MDA-MB-231 cells. In both cell lines the increase of length and double bounds of the fatty acid corresponds to a decreased incorporation. In particular in both cell lines the amount of incorporated DHA in lipid rafts is lower (about a third) compared to the amount of AA (1\% in MCF-7 and $0.37 \%$ in MDA-MB-231 of total PUFA administered).

As shown in Table 2, when both cell lines are treated with n-3 PUFA or n-6 PUFA, the total fatty acid composition of membrane lipid raft changes. The treatment with AA determines a significant increase of this FA from $4.29 \%$ to $13.27 \%$ in MDA-MB-231 cells and from $8.46 \%$ to $20.65 \%$ in MCF7 cells. Also the total n-6 PUFA content is significantly increased. Furthermore the data show also a significant reduction of n-3 PUFA, included EPA, DPA and DHA.

The incubation with EPA induces a significant increase of this FA from $0.74 \%$ to $6.14 \%$ in MDAMB-231 cells and from $0.77 \%$ to $12.91 \%$ in MCF7 cells, and of total n-3 PUFA content. On the contrary we have verified a reduction of total n-6 PUFA, in particular of AA, in both cell lines 
after EPA treatment. Unexpectedly we found an interesting increase also of docosapentaenoic acid (DPA) content, from $0.33 \%$ to $4.60 \%$ in MDA-MB-231and from $0.46 \%$ to $3.59 \%$ in $\mathrm{MCF}-7$ microdomains.

The treatment with DHA causes in both cell lines a significant increase of C22:6 n-3, and of EPA. At the same time, the results show a reduction of AA only in MDA-MB-231 cells.

Worth of note is that the percentage of saturated fatty acids is not changed by PUFA treatment and is higher for MDA-MB-231 (around 70\%) in comparison to MCF-7 (around 45\%).

From these data it is possible to observe that PUFA are all incorporated in cell membrane microdomains even if with different degrees.

\subsection{Changes of fatty acid composition in different phospholipid species of lipid rafts after treatment with n-3 and n-6 PUFA}

To detect if PUFA treatment induces specific changes in fatty acid composition of raft phospholipids, we have purified raft phospholipids and analyzed their fatty acid composition. Table 3 refers to polyunsaturated fatty acid composition of microdomains phospholipids isolated from MDA-MB-231 cells. After AA treatment it is possible to detect an increase of C20:4 n-6 in PI, PS, PC, and SM, while the concentration of AA in PE does not change; also in MCF7 microdomains (see Table 4), the AA treatment determines an enrichment of this FA, especially in PI, PS, PC and SM. The treatment with EPA induces an increase of C20:5 n-3 in all PLs but not in SM in MDA-MB-231 as in MCF7 microdomains. Also the content of DPA, derived from EPA metabolism, increases in PE, PS and PC of MDA-MB-231 rafts, but not in PI and SM, On the contrary in MCF7 rafts EPA improves in all phospholipids. This data is in agreement with Table 1 that indicates an higher incorporation of EPA in phospholipids and raft of MCF-7 cells. Furthermore after EPA treatment the level of DHA, derived from its metabolism, increases in PI of MDA-MB-231 microdomains, while in MCF7 microdomains DHA increases in all PLs except in PI. The data indicate also a reduction of AA, and in general of n-6 PUFA, especially in PI, PS, and PC of MDA-MB-231 microdomains, and in PE and PS in MCF7 rafts. 
The incubation with DHA determines its incorporations in all PLs of both cell lines, a reduction of AA in PE and PI of MDA-MB-231 and in PI of MCF-7 microdomains. Moreover C22:6 n-3 causes a significant increase of EPA in all PLs but in PS of MDA-MB-231 rafts. However this enrichment induces a significant reduction of MUFA and SFA content especially in PI and PC of MDA-MB231 lipid rafts.

\subsection{Effects of PUFA incorporation on sphingomyelin and cholesterol content}

Collected data indicate that n-3 PUFA treatment modifies the content of sphingomyelin (SM). In particular DHA incubation significantly reduces SM in lipid rafts, but not in whole cells. Figure 2 reports the relative content related to controls of SM after PUFA treatment.

In particular in MCF7 microdomains DHA induces a reduction of SM to about 50\% (from 107.53

to $48.51 \mathrm{nmol} / \mathrm{mg}$ Prot) and in MDA-MB-231-microdomains to 60\% (from 244.22 to 162.35 $\mathrm{nmol} / \mathrm{mg}$ Prot.). EPA treatment induces a slight non significant reduction of SM only in MDA-MB231 lipid rafts (see Fig. 2b).

On the contrary the AA treatment does not determine a reduction of this sphingolipid, in microdomains and in whole cells conversely it induces an increase that is not statistically significant due to the high variability of the data (see Fig. 2). We have also studied the effects of PUFA treatment on cholesterol content in breast cancer cell rafts. The data indicate that DHA determines a remarkable and significant reduction of this sterol, especially in MDA-MB-231 (from 829.39 to $585.62 \mathrm{nmol} / \mathrm{mg}$ Prot.) (see Fig. 3b). In MCF7 cells the cholesterol reduction corresponds to -40\%, from 208.88 to $150.28 \mathrm{nmol} / \mathrm{mg}$ Prot. (see Fig. 3a). Worth of note is that the content of both cholesterol and sphingomyelin are higher in MDA-MB-231 cells.

The incubation with EPA determines a slight cholesterol reduction in MDA-MB-231 lipid rafts, to $681.29 \mathrm{nmol} / \mathrm{mg}$ prot. (see Fig. 3b).

Furthermore, the treatment with AA produces, in MDA-MB-231 lipid rafts a significant increase of cholesterol raising the content to 1033.24 nmol/mg Prot (see Fig. 3b).

\subsection{AFM investigation}


Lipid rafts were also analyzed by means of AFM. In particular, AFM investigation allowed to relate chemical-physical modifications, induced in MCF7 and MDA-MB-231 lipid rafts by the DHA incubation, to their morpho-dimensional changes. In Fig. 4, as an example, two AFM topography images of MCF7 lipid rafts before (a) and after (b) DHA incorporation have been reported. The height profiles corresponding to the black lines drawn in Figs. $\mathbf{4 a}$ and $\mathbf{4 b}$ have been showed in Figs. 5a and 5b respectively. After DHA incorporation, AFM images clearly show a reduction of the number of lipid rafts (in the order of about 20-30\%). Moreover, DHA treated lipid rafts show, on average, a lateral size larger than the control ones. In addition, interestingly the profile lines (see Fig. 5) show the presence, in the DHA incorporated rafts, of two different height ranges (4-4.5 $\mathrm{nm}$ and 6-6.5 $\mathrm{nm}$ ). In particular, the $6 \mathrm{~nm}$ thickness of lipid rafts could be produced by DHA incorporation effects. On the contrary, untreated lipid rafts show a constant height of about $4 \mathrm{~nm}$ as awaited for a membrane lipid bilayer. The same results have been obtained on MDA-MB-231 lipid rafts.

Finally, high resolution AFM imaging, obtained visualizing nanometer scan areas, allowed to observe on the lipid raft surface of DHA treated MCF7 cells, pores with an average diameter of about $7 \mathrm{~nm}$ (see Fig. 6a). Although the data do not allow to identify the visualized structures, the pore diameter appears to be in good agreement with the dimensions of proteins observed by AFM on biological membranes. The histogram in Fig. 6b shows the measured values along and perpendicularly the scan direction obtained analysing the height profiles drawn in eleven AFM topography images. In particular, along the scan direction the pore lateral size showed a normal distribution centred on $9.5 \pm 1.2 \mathrm{~nm}($ mean $\pm \mathrm{SD}, \mathrm{n}=34)$ while in the perpendicular direction a normal distribution centred on $7.5 \pm 0.8 \mathrm{~nm}($ mean $\pm \mathrm{SD}, \mathrm{n}=34)$.

\section{DISCUSSION}

Dietary lipids are important for the integrity and functionality of cell membranes. It is recognized that intake of n-3 PUFA can impact numerous processes in the body, including cancer, 
cardiovascular, neurological and immune functions [23-25]. Many studies demonstrated that n-3 PUFA act with synergy to anticancer drugs in cells culture or in tumor-bearing animals, decreasing tumor size, reducing side effects and prolonging survival [26-28].

Lipid rafts are known to be rich in signalling molecules and to regulate signal transduction in normal and cancer cells activating or suppressing phosphorylation cascades related to growth, survival and many other physiological processes, mediated by membrane proteins such as Src, FAK, heterotrimeric G protein subunits and tyrosin kinases receptors as EGFR [29, 30].

The key difference between the lipid rafts and the membrane bilayers from which they are formed is their lipid composition. Rafts contain cholesterol and sphingolipids at concentrations up to $50 \%$ higher than rest of the membrane. The hydrophobic alkyl chains of lipids within the rafts are more saturated compared to the surrounding bilayer and hence tightly packed [31-33].

Our study was aimed to understand if the omega-3 effects on cancer cells may be related to alteration of biochemical and biophysical characteristics of lipid rafts.

We used different long chain PUFA in relative high concentration, due to the fact that in our condition the cells are relatively resistant to fatty acid treatment.

The PUFA concentration of human blood may vary according to age, sex, genetics, diseases and dietary habits [34]. Recent reports from the EPIC-Norfolk cohort study showed that the average concentrations of EPA, DHA in plasma are 56.1 and $236.2 \mu \mathrm{mol} / \mathrm{L}$ respectively with a high individual variability [35].

These data demonstrate that in vivo the doses used in our study can be reached and in some case are relative normal. Moreover observations also from our laboratory indicate very low levels of omega3 PUFA in blood of breast and colon cancer patients [36].

In literature there are different reports about cancer cells treatment with different protocols for PUFA administration and different dosages are reported ranging from 10-200 $\mu \mathrm{M}$ for different times, from 24 hrs to 8 days of treatment. These differences may be due to the fatty acid solubility in different mediums, mixture of PUFA, type of employed reagent and their relative purity (i.e. free 
fatty acid, sodium salt, ethyl esters, TGs, solubilised with serum or precharged on albumin or LDL).

As our goal was to induce fatty acid incorporation within lipid raft; we used fatty acid sodium salts solubilised in medium containing $10 \%$ serum. In these conditions the cells are alive around $80 \%$ after $72 \mathrm{hrs}$ of treatment [20] and were able to incorporate fatty acids, to metabolize them and to insert them into cell membrane lipid rafts, as indicated by present data. The existence of elongases and desaturases able to metabolize PUFA is reported in many tumor cells [37].

In fact our data demonstrate that PUFA and their metabolites are inserted with different yield in cell membrane microdomains and are able to alter fatty acid composition without decreasing the total percentage of saturated fatty acids that characterize these structures. In particular in MDA-MB-231 cells, that displays the highest content of Chol and saturated fatty acids, we observed the lowest incorporation of DHA, probably for sterical reasons; nevertheless DHA was able to decrease Chol and SM content.

Some epidemiological studies addressed the hypothesis that it is possible to correlate breast cancer incidence with Stearoyl-CoA desaturase (SCD-1) expression and activity. Low SCD-1 activity is associated with decreased risk of breast cancer, and it is hypothesized that it can be measured by substrate to product ratios such as the saturation index measured in blood, or plasma or erythrocyte membrane as C16:0/C16:1 or C18:0/C18:1.

For instance in the Italian population [38] it was demonstrated a positive correlation between saturation index and protection from breast cancer.

Interestingly previous results from our lab [20] demonstrated that MCF-7 and MDA-MB231 cell membrane are rich of oleic acid with a very low saturation index around 0.5 .

In this study we isolated lipid raft that, as their nature, are very rich in saturated fatty acids. Nevertheless, while in MDA-MB-231 saturation index of the rafts is around 4, MCF-7 express a saturation index less than 1 with high levels of monounsaturated fatty acids. This may indicate that these cell lines may be different sensible to new therapeutic approaches using SCD-1 inhibitors 
[39]. In fact AA, EPA and DHA increase the saturation index only in MCF-7 cells while low changes are recorded in MDA-MB-231 cells. Moreover omega-6 and omega-3 PUFA may have different regulatory effects on SCD-1 gene expression as already demonstrated in animal models $[40,41]$. Further and finalized experiments are needed to address this issue. We have also analyzed the changes in content of sphingomyelin and cholesterol in breast cancer cell rafts after PUFA treatment. In particular, we have observed that DHA determines a reduction of SM content in lipid rafts of both cell lines.

These reductions might be due to an activation of sphingomyelinase (SMase). SMase is an enzyme that catalyzes the hydrolysis of SM to ceramide. A variety of studies have shown that the ceramide is ubiquitously produced during cellular stress and is associated with apoptosis [42, 43]. The neutral SMase (N-SMase) is a plasma membrane-bound enzyme that is implicated in mediating apoptosis and it resides in lipid rafts [44]. Therefore factors influencing the lipid composition of membranes can influence the activity and distribution of $\mathrm{N}$-SMase in microdomains. Wu et al. already demonstrated that in Jurkat leukemic cells, EPA and DHA increase the N-SMase activity inducing SM hydrolysis [45], our data are in agreement with these observations.

We also showed that DHA, more than EPA, determines a reduction of cholesterol in lipid rafts of breast cancer, especially in MDA-MB-231 cells.

Cholesterol is the dynamic "glue" that holds the rafts together. Due to the rigid nature of the sterol group, cholesterol partitions preferentially into the lipid rafts where acyl chains of the lipids tend to be more rigid and in a less fluid state. It should be noted that cholesterol has the ability to pack in between the lipids in microdomains, serving as a molecular spacer and filling any voids between associated sphingolipids. It therefore plays a very important and dominant role in raft formation and stabilization $[9,31,46]$. Besides, cholesterol is known to play a important role in maintaining membrane integrity, trafficking, signal transduction and fluidity [31, 32]. Cholesterol depletion results in the disorganization of lipid raft microdomains and also in the dissociation of proteins [47] that are bound to the lipid raft. Cholesterol accumulation is known to be associated in many tumors, 
including prostate cancer and oral cancer [48, 49], and dysregulated in lung and breast cancers [50, 51]. Our data indicating that MDA-MB-231, that is highly aggressive tumor type representative, has an higher concentration of cholesterol in rafts and resistance to PUFA incorporation are in line to above cited reports. Li et al. demonstrated also that cholesterol depletion down regulate phosphoAkt and phospho-ERK, which play critical roles in cell survival and induce apoptosis [52].

A growing number of experimental observations demonstrates the incompatibility between cholesterol and DHA [53-55]. Saturated fatty acids, compared to PUFA, have a preferential affinity for cholesterol. This sterol condenses monolayers made from phospholipids containing a saturated chain in the sn-1 position and a saturated, oleic or $\alpha$-linoleic, but not DHA, in the sn-2 position. This relationship provides the basis for a lipid-driven mechanism for the lateral segregation of membrane elements into cholesterol-rich and -poor microdomains. For example, unfavourable interaction between cholesterol and PUFA chains has been clearly demonstrated by the exclusion of cholesterol from dipolyunsaturated phosphatidylcholine (PC) liposomes where it is forced to directly contact polyunsaturated chains. Studies using a variety of techniques including differential scanning calorimetry [56], ${ }^{1} \mathrm{H}$ NMR and nuclear Overhauser enhancement spectroscopy with magic angle spinning, determination of partition coefficients [57], measurements of lateral compressibility [58], and fluorescence anisotropy [59], indicate that the poor affinity of DHA for cholesterol provides a lipid-driven mechanism for lateral phase separation of cholesterol-rich lipid microdomains from the surrounding bulk membrane. This could in principle alter the size, stability and distribution of cell surface lipid microdomains such as rafts. Indeed, evidences obtained from model membrane suggest that the energetically less favourable interaction between cholesterol and PUFA, especially DHA, promotes lateral phase segregation into sterol-poor/ PUFA-rich and sterolrich/saturated fatty acid-rich microdomains [60-62].

Since lipid rafts are predominantly enriched in saturated fatty acids-containing sphingolipid and cholesterol, the incorporation of PUFA, especially DHA, determines in breast cancer cells a disruption of lipid rafts and a formation of the PUFA rich/cholesterol-poor domains. 
On the contrary in our data AA increases cholesterol in MDA-MB-231 together with a slight increase of SM. Recently Pike and collaborators have demonstrated that lipid rafts are enriched in AA utilizing ESI/MS analysis [63]. This fatty acid is mainly linked to PE and PL-PE (plasmalogens) that are mainly in the inner membrane leaflet, while cholesterol and sphingomyelin are mainly in the outer leaflet. They speculate that this may represent a pool of AA for the generation of free AA in response to cell activation and subsequently increase of cell mediators such as PGE2.

Our data on breast cancer cells are in agreement as AA represents the major PUFA of PLs in lipid rafts and is particularly concentrated in PE.

The chemical physical features of fatty acids and related phospholipids are mainly influenced by their length and double bounds presence. Little variations may greatly influence their ability to interact in membranes with other lipids such as cholesterol. For instance there are many differences between phospholipids containing DHA (22:6) and DPA (22:5); the DHA chain with one additional double bond is more flexible at the methyl end and isomerizes with shorter correlation times. The loss of a single double bond from DHA to DPA results in a more even distribution of chain densities along the normal bilayer [64]. The different effects of DHA, EPA and AA integrated in raft phospholipids on lipid content may be explained by their relative structural differences.

A recent review of Shaikh [65] has hypothesized a new model n-3 PUFA raft interaction with the formation of "declustered rafts". He raised also many question about the direct or indirect effects of n-3 PUFA on rafts. Our results demonstrate the direct incorporation of PUFA in phospholipids of detergent resistant membranes isolated with Triton $\mathrm{X}-100$; when DHA is incorporated into raft it may induce the formation of what they define "declustered rafts" with reorganization of lipids and proteins. Preliminary results from our lab also demonstrate that DHA displaces EGFR from MDAMB-231 microdomains.

In literature data on the synchronous effects of tamoxifen and n-3 PUFA are reported, pointing out the in vitro and in vivo capacity of omega-3, or their metabolites, to reduce cellular proliferation and 
increase apoptosis. One of the mechanisms of action attributed to the apoptosis augment in DHA/EPA-treated MDA-MB-231 cells was impairment of Akt phosphorylation and NF-kB activity [66]. In line with this, in vivo studies showed an increased apoptotic index of MCF-7 cells injected into flaxseed oil-fed nude mice. This reduction was probably due to the down regulation of tyrosine kinase receptors such as EGFR and HER2, and the subsequent down regulation of Akt [67]. Interestingly some paper also reported the ability of tamoxifen to induce changes in physical and chemical properties of cell membrane inducing also changes in lipid composition and decreasing cholesterol content [68]. Our data suggest that DHA may act synergistically in this sense with tamoxifen.

AFM analysis allowed us to characterize quantitatively morpho-dimensional changes induced by DHA incorporation in lipid rafts. In particular, for both the two cell lines (MCF-7 and MDA-MB231) the DHA treatment produced a decrease of the lipid rafts in the order of about $20 \%-30 \%$ (see Fig. 4). It is worth noticing that after DHA incorporation lipid rafts exhibit two different height ranges. In fact, some lipid rafts show an height of 4-4.5 $\mathrm{nm}$ such as the untreated ones, while several lipid rafts, in the order of about $20 \%$, have a higher height of 6-6.5 nm (see Fig. 5).

These changes might influence resident protein conformation turning on and/or off signalling proteins, and modulate cellular events. In fact acyl moieties of proteins partition in the cytoplasmic leaflet of the membrane lipid bilayer. Lipid alterations in the cytoplasmic lipid leaflet of membrane rafts would hence be particularly effective in displacing palmitoylated proteins.

High resolution AFM imaging showed on MCF-7 lipid rafts, after DHA incorporation, pores in agreement, for dimensions and shape, with membrane proteins (see Fig. 6). A more accurate investigation, planned in the next future, and carried out using specific antibodies could confirm the nature of the observed structures and allow their identification. These preliminary results suggest that AFM could be an useful tool to characterize changes in the membrane protein content induced by n-3 PUFA treatment at single protein level.

The data presented show an incorporation of PUFA with different specificity for the PL moiety. 
This may be relevant to the formation of PUFA metabolites (prostaglandins, prostacyclins, leukotrienes, resolvines and protectines) of phospholipids deriving second messengers and signal transduction activation.

As an example phosphatidylinositol and phosphatidylcholine may act as sources of intracellular signals in response to extracellular signals which interact with receptors on the outer layer of plasma membrane. Hormones, or other extracellular signals, bind to the plasma membrane receptor which leads to activation of phospholipase C. Phospholipase C hydrolyses PI 4,5bisphosphate(PIP2) releasing inositol 1,4,5-triphosphate (IP3) and diacylglycerol (DG). In consequence, IP3 (which is water soluble) causes release of calcium $(\mathrm{Ca} 2+)$ from the endoplasmic reticulum. The combination of DG (which is lipid soluble and stays bound to the plasma membrane) and increased levels of $\mathrm{Ca} 2+$, together with membrane PS, lead to activation of the enzyme protein kinase $\mathrm{C}(\mathrm{PKC})$. This enzyme catalyses the phosphorylation of some cellular proteins, affecting cellular responses to the initial extracellular trigger. Fatty acids originating from the sn-2 glycerol carbon of PC greatly influence the activity of second messengers (DG). In this view the changes demonstrated in this paper with incorporation of fatty acids in PC, PS and PI phospholipids may contribute to explain the overall effects of PUFA reducing cell growth of breast cancer cells. Interestingly the treatment with n-3 PUFA decrease the content and change the fatty acid composition of PIP2 in Fat-1 CD4+ T-cells [69].

Moreover, EPA and DHA increasing the unsaturated state of phospholipids in lipid rafts of breast cancer cells, alter their physical-chemical properties. Many acylated proteins directly interact with membrane lipid bilayers by their saturated acyl moieties. Then we suggest that altered lipid composition of microdomains might determine the displacement of proteins from lipid rafts in $n-3$ PUFA-treated cells with alteration of signal transduction

In conclusion n-3 PUFA are able to modify lipid raft biochemical and biophysical features leading to decrease of breast cancer cell proliferation probably through different mechanisms related to acyl chain length and unsaturation. While EPA may contribute to cell apoptosis mainly through decrease 
of AA concentration in lipid raft phospholipids, indicating its influence in production of second messengers and PUFA metabolites, DHA may change the biophysical properties of lipid rafts decreasing the content of cholesterol and probably a redistribution of key proteins such as EGFR, ER- $\alpha$ and RAS. Future research will be dedicate to asses these hypotheses.

\section{Acknowledgements}

This paper is dedicated to the memory of Prof. Bruno Berra. We are grateful to anonymous reviewers for their constructive suggestions.

Financial support to Dr Angela M. Rizzo came from Italian Space Agency (ASI).

INDEX ENTRIES: EPA, DHA, AA, PUFA, breast cancer, lipid rafts, phospholipids, membrane.

\section{Conflict of Interest}

None declared 


\section{Bibliography:}

[1] Bagga D, Anders KH, Wang HJ, Glaspy JA (2002) Long-chain n-3-to-n-6 polyunsaturated fatty acid ratios in breast adipose tissue from women with and without breast cancer. Nutr Cancer. 2002. 42(2), 180-5.

[2] Manni A, Xu H, Washington S, Aliaga C, Cooper T, Richie JP Jr, Bruggeman R, Prokopczyk B, Calcagnotto A, Trushin N, Mauger D, Verderame MF, El-Bayoumy K (2010) The impact of fish oil on the chemopreventive efficacy of tamoxifen against development of N-methyl-N-nitrosoureainduced rat mammary carcinogenesis. Cancer Prev Res (Phila). 2010. 3(3), 322-30.

[3] Gillet L, Roger S, Bougnoux P, Le Guennec JY, Besson P (2011) Beneficial effects of omega-3 long-chain fatty acids in breast cancer and cardiovascular diseases: voltage-gated sodium channels as a common feature?. Biochimie. 2011.93(1), 4-6.

[4] Olbrich K, Rawicz W, Needham D, Evans E (2000) Water permeability and mechanical strength of polyunsaturated lipid bilayers. Biophys J. 2000. 79(1), 321-7.

[5] Rawicz W, Olbrich KC, McIntosh T, Needham D, Evans E (2000) Effect of chain length and unsaturation on elasticity of lipid bilayers. Biophys J. 2000. 79(1), 328-39.

[6] Brzustowicz MR, Cherezov V, Zerouga M, Caffrey M, Stillwell W, Wassall SR (2002) Controlling membrane cholesterol content. A role for polyunsaturated (docosahexaenoate) phospholipids. Biochemistry. 2002. 41(41), 12509-19.

[7] Botelho AV, Gibson NJ, Thurmond RL, Wang Y, Brown MF (2002) Conformational energetics of rhodopsin modulated by nonlamellar-forming lipids. Biochemistry. 2002. 41(20), 6354-68.

[8] Mitchell DC, Niu SL, Litman BJ (2003) Enhancement of G protein-coupled signaling by DHA phospholipids. Lipids. 2003. 38(4), 437-43.

[9] London E, Brown DA (2000) Insolubility of lipids in triton X-100: physical origin and relationship to sphingolipid/cholesterol membrane domains (rafts). Biochim Biophys Acta. 2000. 1508(1-2), 182-95.

[10] Pike LJ (2004) Lipid rafts: heterogeneity on the high seas. Biochem J. 2004. 378(Pt 2), 281-92. 
[11] Lingwood D, Simons K (2007) Detergent resistance as a tool in membrane research. Nat Protoc. 2007. 2(9), 2159-65.

[12] Simons K, Toomre D (2001) Lipid rafts and signal transduction. Nat Rev Mol Cell Biol. 2000. 1(1), 31-9. Erratum in: Nat Rev Mol Cell Biol. 2001. 2(3), 216.

[13] Razani B, Woodman SE, Lisanti MP (2002) Caveolae: from cell biology to animal physiology. Pharmacol Rev. 2002. 54(3), 431-67.

[14] van Meer G (2002) Cell biology. The different hues of lipid rafts. Science. 2002 296(5569), 855-7.

[15] Fastenberg ME, Shogomori H, Xu X, Brown DA, London E (2003) Exclusion of a transmembrane-type peptide from ordered-lipid domains (rafts) detected by fluorescence quenching: extension of quenching analysis to account for the effects of domain size and domain boundaries. Biochemistry. 2003. 42(42), 12376-90.

[16] Gimpl G, Burger K, Fahrenholz F (1997) Cholesterol as modulator of receptor function. Biochemistry. 1997. 36(36), 10959-74.

[17] Pike LJ, Casey L (2002) Cholesterol levels modulate EGF receptor-mediated signaling by altering receptor function and trafficking. Biochemistry. 2002. 41(32), 10315-22.

[18] Hanzal-Bayer MF, Hancock JF (2007) Lipid rafts and membrane traffic. FEBS Lett. 2007. 581(11), 2098-104.

[19] Jury EC, Flores-Borja F, Kabouridis PS (2007) Lipid rafts in T cell signalling and disease. Semin Cell Dev Biol. 2007. 18(5), 608-15.

[20] Corsetto PA, Montorfano G, Zava S, Jovenitti IE, Cremona A, Berra B, Rizzo AM, Effects of n-3 PUFAs on breast cancer cells through their incorporation in plasma membrane. Lipids Health Dis. 2011. 10, 73.

[21] Fabelo N, Martin V, Santpere G, Marìn R, Torrent L, Ferrer I, Dìaz M, (2011) Severe alterations in lipid composition of frontal cortex lipid rafts from Parkinson's disease and incidental Parkinson's disease. Mol Med 17(9-10), 1107-1118. 
[22] Lowry OH, Rosebrough NJ, Farr AL, Randall RJ (1951) Protein measurement with the Folin phenol reagent. J Biol Chem 1951. 193(1), 265-275.

[23] Jho DH, Cole SM, Lee EM, Espat NJ (2004) Role of omega-3 fatty acid supplementation in inflammation and malignancy. Integr Cancer Ther. 2004. 3(2), 98-111.

[24] Innis SM, Jacobson K (2007) Dietary lipids in early development and intestinal inflammatory disease. Nutr Rev. 2007. 65(12 Pt 2), S188-93.

[25] Calder PC (2006) n-3 polyunsaturated fatty acids, inflammation, and inflammatory diseases. Am J Clin Nutr. 2006. 83(6 Suppl), 1505S-1519S.

[26] Menendez JA, Lupu R, Colomer R (2005) Exogenous supplementation with omega-3 polyunsaturated fatty acid docosahexaenoic acid (DHA; 22: 6n-3) synergistically enhances taxane cytotoxicity and downregulates Her-2/neu (c-erbB-2) oncogene expression in human breast cancer cells. Eur J Cancer Prev. 2005. 14, 263-70.

[27] Calviello G, Di NF, Serini S, Piccioni E, Boninsegna A, Maggiano N (2005) Docosahexaenoic acid enhances the susceptibility of human colorectal cancer cells to 5-fluorouracil. Cancer Chemother Pharmacol. 2005. 55, 12-20.

[28] Lindskog M, Gleissman H, Ponthan F, Castro J, Kogner P, Johnsen JI (2006) Neuroblastoma cell death in response to docosahexaenoic acid: sensitization to chemotherapy and arsenic-induced oxidative stress. Int J Cancer. 2006.118, 2584-93.

[29] Edidin M (2001) Membrane cholesterol, protein phosphorylation, and lipid rafts. Sci STKE. 2001. 67, E1.

[30] Zajchowski LD, Robbins SM (2002) Lipid rafts and little caves. Compartmentalized signalling in membrane microdomains. Eur J Biochem. 2002. 269, 737-752.

[31] Epand RM (2008) Proteins and cholesterol-rich domains. Biochim Biophys Acta. 2008. 1778(7-8), 1576-82.

[32] Maxfield F, Tabas I (2005) Role of cholesterol and lipid organization in disease. Nature. 2005. 
$438,612-621$.

[33] Silvius JR (2003) Role of cholesterol in lipid raft formation: lessons from lipid model systems. Biochim Biophys Acta. 2003. 1610, 174-183.

[34] Rizzo AM, Montorfano G, Negroni M, Adorni L, Berselli P, Corsetto P, Wahle K, Berra B (2010) A rapid method for determining arachidonic:eicosapentaenoic acid ratios in whole blood lipids: correlation with erythrocyte membrane ratios and validation in a large Italian population of various ages and pathologies. Lipids Health Dis. 2010. 27, 9-7.

[35] Welch AA, Shakya-Shrestha S, Lentjes MA, Wareham NJ, Khaw KT (2010) Dietary intake and status of n-3 polyunsaturated fatty acids in a population of fish-eating and non-fish-eating meateaters, vegetarians, and vegans and the product-precursor ratio [corrected] of $\alpha$-linolenic acid to long-chain n-3 polyunsaturated fatty acids: results from the EPIC-Norfolk cohort. Am J Clin Nutr. 2010. 92(5), 1040-51.

[36] Rizzo AM, Montorfano G, Fallini M, Negroni M, Paleari D, Berra B, Garassino MC. (2006). A case-control study in cancer patients (PTS): The arachidonic acid/eicosapentaenoic acid (AA/EPA) ratio as a biomarker. Annnals of oncology 2006. 17, 306-306.

[37] Hrelia S, Bordoni A, Biagi P, Rossi CA, Bernardi L, Horrobin DF, Pession A (1996). gammaLinolenic acid supplementation can affect cancer cell proliferation via modification of fatty acid composition. Biochem Biophys Res Commun. 1996. 225, 441-7.

[38] Pala V, Krogh V, Muti P, Chajès V, Riboli E, Micheli A, Saadatian M, Sieri S, Berrino F. (2001) Erythrocyte membrane fatty acids and subsequent breast cancer: a prospective Italian study. J Natl Cancer Inst. 2001. 18;93(14), 1088-95.

[39] Mason P, Liang B, Li L, Fremgen T, Murphy E, Quinn A, Madden SL, Biemann HP, Wang B, Cohen A, Komarnitsky S, Jancsics K, Hirth B, Cooper CG, Lee E, Wilson S, Krumbholz R, Schmid S, Xiang Y, Booker M, Lillie J, Carter K. (2012) SCD1 Inhibition Causes Cancer Cell Death by Depleting Mono-Unsaturated Fatty Acids. PLoS One. 2012. 7(3):e33823. 
[40] Yang ZH, Miyahara H, Takemura S, Hatanaka A. (2011) Dietary saury oil reduces hyperglycemia and hyperlipidemia in diabetic KKAy mice and in diet-induced obese C57BL/6J mice by altering gene expression. Lipids. 2011. 46(5):425-34.

[41] Muhlhausler BS, Cook-Johnson R, James M, Miljkovic D, Duthoit E, Gibson R (2010). Opposing effects of omega-3 and omega- 6 long chain polyunsaturated Fatty acids on the expression of lipogenic genes in omental and retroperitoneal adipose depots in the rat. J Nutr Metab. 2010. vol. 2010, Article ID 927836, 9 pages, doi:10.1155/2010/927836

[42] Tonnetti L, Verí MC, Bonvini E, D'Adamio L (1999) A role for neutral sphingomyelinasemediated ceramide production in $\mathrm{T}$ cell receptor-induced apoptosis and mitogen-activated protein kinase-mediated signal transduction. J Exp Med. 1999. 189(10), 1581-9.

[43] Jayadev S, Liu B, Bielawska AE, Lee JY, Nazaire F, Pushkareva MYu, Obeid LM, Hannun YA (1995) Role for ceramide in cell cycle arrest. J Biol Chem. 1995. 270(5), 2047-52.

[44] Veldman RJ, Maestre N, Aduib OM, Medin JA, Salvayre R, Levade T (2001) A neutral sphingomyelinase resides in sphingolipid-enriched microdomains and is inhibited by the caveolinscaffolding domain: potential implications in tumour necrosis factor signaling. Biochem J. 2001. 355(Pt 3), 859-68.

[45] Wu M, Harvey KA, Ruzmetov N, Welch ZR, Sech L, Jackson K, Stillwell W, Zaloga GP, Siddiqui RA (2005) Omega-3 polyunsaturated fatty acids attenuate breast cancer growth through activation of a neutral sphingomyelinase-mediated pathway. Int J Cancer. 2005. 117(3), 340-8.

[46] Wang TY, Silvius JR (2001) Cholesterol does not induce segregation of liquid-ordered domains in bilayers modeling the inner leaflet of the plasma membrane. Biophys J. 2001. 81(5), $2762-73$

[47] Barman S, Nayak DP (2007) Lipid raft disruption by cholesterol depletion enhances influenza [48] Freeman MR, Solomon KR (2004) Cholesterol and prostate cancer. J Cell Biochem. 2004. 91, 54-69. 
[49] Kolanjiappan K, Ramachandran CR, Manoharan S (2003) Biochemical changes in tumor tissues of oral cancer patients. Clin Biochem. 2003. 36, 61-65.

[50] Bennis F, Favre G, Le GF, Soula G (1993) Importance of mevalonate-derived products in the control of HMG-CoA reductase activity and growth of human lung adenocarcinoma cell line A549. Int J Cancer. 1993. 55, 640-645.

[51] El-Sohemy A, Archer MC (2000) Inhibition of N-methyl-N-nitrosourea-and 7,12dimethylbenz[a] anthracene-induced rat mammary tumorigenesis by dietary cholesterol is independent of Ha-Ras mutations. Carcinogenesis. 2000. 21, 827-831.

[52] Li YC, Park MJ, Ye SK, Kim CW, Kim YN (2006) Elevated levels of cholesterol-rich lipid rafts in cancer cells are correlated with apoptosis sensitivity induced by cholesterol-depleting agents. Am J Pathol. 2006. 168, 1107-1118.[53] Huster D, Arnold K, Gawrisch K (1998) Influence of docosahexaenoic acid and cholesterol on lateral lipid organization in phospholipid mixtures. Biochemistry. 1998. 37, 17299-17308.

[54] Mitchell DC, Litman BJ (1998) Effect of cholesterol on molecular order and dynamics in highly polyunsaturated phospholipid bilayers. Biophys J. 1998. 75, 896-908.

[55] Niu SL, Litman BJ (2002) Determination of membrane cholesterol partition coefficient using a lipid vesicle cyclodextrin binary system: effect of phospholipid acyl chain unsaturation and headgroup composition. Biophys J. 2002. 83, 3408-3415.

[56] Zerouga M, Jenski LJ, Stillwell W (1995) Comparison of phosphatidylcholines containing one or two docosahexaenoic acyl chains on properties of phospholipid monolayers and bilayers. Biochim Biophys Acta. 1995. 1236, 266-272.

[57] Kariel N, Davidson E, Keough KM (1991) Cholesterol does not remove the gel-liquid crystalline phase transition of phosphatidylcholines containing two polyenoic acyl chains. Biochim Biophys Acta. 1991. 1062, 70-76.

[58] Needham D, Nunn RS (1990) Elastic deformation and failure of lipid bilayer membranes containing cholesterol. Biophys J. 1990. 58, 997-1009. 
[59] Mitchell DC, Litman BJ (1998) Molecular order and dynamics in bilayers consisting of highly polyunsaturated phospholipids. Biophys J. 1998. 74, 879-891.

[60] Brzustowicz MR, Cherezov V, Caffrey M, Stillwell W, Wassall SR (2002) Molecular organization of cholesterol in polyunsaturated membranes: microdomain formation. Biophys $\mathrm{J}$. 2002. 82, 285-298.

[61] Brzustowicz MR, Cherezov V, Zerouga M, Caffrey M, Stillwell W, Wassall SR (2002) Controlling membrane cholesterol content. A role for polyunsaturated (docosahexaenoate) phospholipids. Biochemistry. 2002. 41, 12509-12519.

[62] Pasenkiewicz-Gierula M, Subczynski WK, Kusumi A (1990) Rotational diffusion of a steroid molecule in phosphatidylcholine-cholesterol membranes: fluid-phase microimmiscibility in unsaturated phosphatidylcholine-cholesterol membranes. Biochemistry. 1990. 29, 4059-4069.

[63] Pike LJ, Han X, Chung KN, Gross RW.(2002) Lipid rafts are enriched in arachidonic acid and plasmenylethanolamine and their composition is independent of caveolin-1 expression: a quantitative electrospray ionization/mass spectrometric analysis. Biochemistry. 2002. 41(6):207588.

[64] Eldho NV, Feller SE, Tristram-Nagle S, Polozov IV, Gawrisch K. (2003) Polyunsaturated docosahexaenoic vs docosapentaenoic acid-differences in lipid matrix properties from the loss of one double bond. J Am Chem Soc. 2003. 125(21), 6409-21.

[65] Shaikh SR. (2012) Biophysical and biochemical mechanisms by which dietary N-3 polyunsaturated fatty acids from fish oil disrupt membrane lipid rafts. J Nutr Biochem. 2012. 23(2), $101-5$.

[66] DeGraffenried LA, Friedrichs WE, Fulcher L, Fernandes G, Silva JM, Peralba JM, Hidalgo M. (2003).Eicosapentaenoic acid restores tamoxifen sensitivity in breast cancer cells with high Akt activity. Ann Oncol. 2003. 14, 1051-6.

[67] Truan JS, Chen JM, Thompson LU (2010). Flaxseed oil reduces the growth of human breast tumors (MCF-7) at high levels of circulating estrogen. Mol. Nutr. Food Res. 2010. 54, 1414-1421. 
[68] Engelke M, Tykhonova S, Zorn-Kruppa M, Diehl H (2002). Tamoxifen induces changes in the lipid composition of the retinal pigment epithelium cell line D407.Pharmacol Toxicol. 2002. 91, 1321.

[69] Hou TY, Monk JM, Fan YY, Barhoumi R, Chen YQ, Rivera GM, McMurray DN, Chapkin RS (2012). N-3 polyunsaturated fatty acids suppress phosphatidylinositol 4,5-bisphosphate-dependent actin remodelling during CD4+ T-cell activation. Biochem J. 2012. 443, 27-37. 


\section{Figure Legends}

\section{Figure 1- Characterization of Lipid Rafts from human breast cancer cells}

Post-nuclear homogenates from breast cancer cells were extracted using $1 \%$ of Triton X-100 and fractionated in 5-35\% sucrose gradient as described in Methods. The purification of lipid rafts was demonstrated by the enrichment of sphingomyelin, cholesterol and gangliosideby HPTLC, in all fractions isolated from MDA-MB-231 (top) and MCF-7 (bottom) control cells (a,e) and treated with AA (b,f), EPA (c,g), DHA (d,h). Standards were spotted on the same plate (left).

Equal amount of each fractions were subjected to western blot analyses against raft protein marker (flotillin-1) and non raft protein marker (clathrin).

\section{Figure 2- Effects of PUFA on sphingomyelin content of breast cancer cells lipid rafts}

Sphingomyelin content in lipid rafts of MCF7 (top) and MDA-MB-231 (bottom) breast cancer cells is obtained by HPLC-ELSD analysis. Data are represented as percentage of control $\pm S D(n=3)$.

Control cells are not exposed to any exogenous fatty acid but incubated in the same conditions with the same ethanol concentration of treated cells.

Vs respective CTR: * $\mathrm{p}<0.05 ; * * \mathrm{p}<0.01$

\section{Figure 3- Effects of PUFA on cholesterol content of breast cancer cells}

Whole cell cholesterol and cholesterol in lipid rafts of breast cancer MCF7 (top) and MDA-MB-231 (bottom) cells fractionated on sucrose gradient have been analysed by HPLC-ELSD. Cells were treated with AA (C20:4), EPA (C20:5), and DHA (C22:6) for 72hs. Measurements were performed in triplicate and data are presented as percentage of control $\pm \mathrm{SD}(\mathrm{n}=3)$. Control cells are not exposed to any exogenous fatty acids but incubated in the same conditions with the same ethanol concentration of treated cells

Vs respective CTR: * $\mathrm{p}<0.05 ; * * \mathrm{p}<0.01$ 


\section{Figure 4- AFM topographs before and after DHA treatment of MCF7 lipid rafts}

AFM topography images collected in contact mode in buffer solution of MCF7 lipid rafts before (a) and after (b) treatment with DHA (C22:6). Z range (from darkest to lightest): $50 \mathrm{~nm}$. Scan area: $10 \times 10 \mu \mathrm{m}^{2}$.

\section{Figure 5- Height profiles before and after DHA treatment of MCF7 lipid rafts}

Height profiles corresponding to the two black lines drawn in the AFM topography images reported in Fig 3. The DHA incorporated rafts (b) show two different height ranges: 4-4.5 nm and 6-6.5 nm (red arrows) while the control rafts (a) exhibit a constant height of about $4 \mathrm{~nm}$ (red arrows). While in the control samples lipid rafts exhibit a constant height of about $4 \mathrm{~nm}$ (see red arrows in a) as awaited for a membrane lipid bilayer, in DHA treated samples, lipid rafts show also the presence of a higher thickness (about $6 \mathrm{~nm}$, see red arrows in b) likely induced by DHA incorporation effects.

\section{Figure 6- High resolution AFM topography}

(a) High resolution AFM topography image (bottom) obtained visualizing a small region (300x300 $\mathrm{nm}^{2}$ ) on the surface of a lipid raft of DHA treated MCF7 cells (top). Several pores with a diameter of about $9 \mathrm{~nm}$ (red arrows in the height profile) are clearly visible on the lipid raft surface. (b) Histogram of the lateral dimensions of the membrane pores visualized on MCF7 lipid rafts after DHA treatment. The dimensions have been measured along (horizontal) and perpendicularly (vertical) to the scan direction. The diameters of the visualized pores appear to be in good agreement with the dimensions of membrane proteins observed by AFM. 
